\title{
Paeonol protects rat vascular endothelial cells from ox-LDL-induced injury in vitro via downregulating microRNA-21 expression and TNF- $\alpha$ release
}

\author{
Ya-rong LIU ${ }^{1}$, Jun-jun $\mathrm{CHEN}^{2}$, Min DAl ${ }^{3, *}$ \\ ${ }^{1}$ Key Laboratory of Chinese Medicine Research and Development, ${ }^{2}$ Key Laboratory of Xin'an Medicine, Hefei 230038, China; ${ }^{3}$ Depart- \\ ment of Pharmacy, Anhui University of Traditional Chinese Medicine, Hefei 230031, China
}

Aim: Paeonol (2'-hydroxy-4'-methoxyacetophenone) from Cortex moutan root is a potential therapeutic agent for atherosclerosis. This study sought to investigate the mechanisms underlying anti-inflammatory effects of paeonol in rat vascular endothelial cells (VECs) in vitro.

Methods: VECs were isolated from rat thoracic aortas. The cells were pretreated with paeonol for $24 \mathrm{~h}$, and then stimulated with ox-LDL for another $24 \mathrm{~h}$. The expression of microRNA-21 (miR-21) and PTEN in VECs was analyzed using qRT-PCR. The expression of PTEN protein was detected by Western blotting. TNF- $\alpha$ release by VECs was measured by ELISA.

Results: Ox-LDL treatment inhibited VEC growth in dose- and time-dependent manners (the value of $\mathrm{IC}_{50}$ was about $20 \mathrm{mg} / \mathrm{L}$ at $24 \mathrm{~h}$ ). Furthermore, ox-LDL (20 mg/L) significantly increased miR-21 expression and inhibited the expression of PTEN, one of downstream target genes of miR-21 in VECs. In addition, ox-LDL $(20 \mathrm{mg} / \mathrm{L})$ significantly increased the release of TNF- $\alpha$ from VECs. Pretreatment with paeonol increased the survival rate of ox-LDL-treated VECs in dose- and time-dependent manners. Moreover, paeonol (120 $\mu$ mol/ L) prevented ox-LDL-induced increases in miR-21 expression and TNF- $\alpha$ release, and ox-LDL-induced inhibition in PTEN expression. A dual-luciferase reporter assay showed that miR-21 bound directly to PTEN's 3'-UTR, thus inhibiting PTEN expression. In ox-LDL treated VECs, transfection with a miR-21 mimic significantly increased miR-21 expression and inhibited PTEN expression, and attenuated the protective effects of paeonol pretreatment, whereas transfection with an miR-21 inhibitor significantly decreased miR-21 expression and increased PTEN expression, thus enhanced the protective effects of paeonol pretreatment.

Conclusion: miR-21 is an important target of paeonol for its protective effects against ox-LDL-induced VEC injury, which may play critical roles in development of atherosclerosis.

Keywords: paeonol; atherosclerosis; microRNA-21; PTEN; vascular endothelial cells; oxidized low density lipoprotein; inflammatory reaction

Acta Pharmacologica Sinica (2014) 35: 483-488; doi: 10.1038/aps.2013.190; published online 24 Feb 2014

\section{Introduction}

Atherosclerosis (AS) is a leading cause of morbidity and mortality worldwide and involves multiple genes and other factors $^{[1]}$. Dysfunction of and injury to vascular endothelial cells (VECs) are the driving forces in the initiation and development of AS, whereas ox-LDL is a well-recognized risk factor for VEC damage ${ }^{[2]}$. Paeonol (2'-hydroxy-4'-methoxyacetophenone, $\mathrm{C}_{9} \mathrm{H}_{10} \mathrm{O}_{3}$ ) is one of the main active compounds in Cortex moutan root, which has been used in traditional Chinese medicine for its anti-inflammatory, anti-thrombotic and antioxidant properties ${ }^{[3,4]}$ (Figure 1). A large number of studies have demonstrated that paeonol is effective for preventing and

\footnotetext{
* To whom correspondence should be addressed.

E-mail daiminliao@163.com

Received 2013-08-05 Accepted 2013-12-12
}

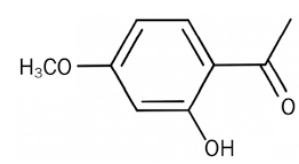

Figure 1. The chemical structure of paeonol from Cortex Moutan.

treating of AS in experimental models by modulating inflammatory reactions during the initiation and development of AS. For example, paeonol inhibits tumor necrosis factor (TNF)-ainduced vascular cell adhesion molecule-1 (VCAM-1) expression, which reduces monocyte adhesion to $\mathrm{VECs}^{[5]}$. Moreover, paeonol suppresses lipopolysaccharide-induced inflammatory cytokine release from macrophage cells and protects mice from lethal endotoxin shock ${ }^{[6]}$. Although the anti-inflamma- 
tory effect of paeonol is well recognized, the exact mechanisms are still unclear.

MicroRNAs (miRNAs) constitute a broad class of short noncoding RNAs that have been extensively studied in the context of cancer. Recently, increasing evidence has indicated that miRNAs are involved in the development of $\mathrm{AS}^{[7]}$. miRNAs can modulate the expression of various cytokines or inflammatory molecules by binding to the complementary target sites in the $3^{\prime}$ untranslated regions ( $\left.3^{\prime} \mathrm{UTR}\right)$ of mRNA sequences and inducing mRNA degradation or translational repression. MicroRNA-21 (miR-21) is selectively expressed in vascular cells and modulates proliferation, invasion, apoptosis and inflammation ${ }^{[8]}$. In the peripheral arterial system, miR21 is upregulated after acute vascular injury, and knockdown of miR-21 has been shown to reduce neointima formation ${ }^{[9]}$. Therefore, we were interested in determining whether miR-21 is involved in modulating the therapeutic effect of paeonol on AS, as well as the downstream target of miR-21 in VECs.

In this study, we investigated the mechanisms underlying the protective effect of paeonol in a VEC injury model. We assessed the expression of miR-21 and its downstream target genes, such as PTEN, in injured VECs in the presence or absence of paeonol treatment .

\section{Materials and methods \\ Animals}

Healthy male Sprague-Dawley rats weighing $150-170 \mathrm{~g}$ were provided by the Experimental Animal Center of Anhui Medical University (Hefei, China). The animal experiments were approved by the local institutional Animal Care and Use Committee.

\section{Chemicals}

Paeonol (98\% purity) was obtained from Baicao Plants Biotech Co, Ltd (Anhui, China). The cell culture materials and fetal bovine serum (FBS) were obtained from GIBCO BRL Co, Ltd (Gaithersburg, USA). Ox-LDL (YB-002-1) was purchased from Yiyuan Biotechnologies Co, Ltd (China). HiPerFect transfection reagent, miR-21 inhibitor and mimic, MiRNeasy Mini Kit (Cat.217004) and MiScript PCR Starter Kit (Cat.218193) were purchased from Qiagen (Germany). The dual-luciferase reporter system (Cat.E1910) was purchased from Promega (USA). The BCA Protein Assay kit was obtained from Shanghai Haoran Bio Technologies Co, Ltd (China). Antibodies against $\beta$-actin and PTEN were purchased from Bioworld Technology Co, Ltd (USA).

\section{Isolation and culture of VECs}

VECs were isolated from rat thoracic aortas as previously described $^{[10]}$. In short, thoracic aortas isolated from SpragueDawley rats were turned over to expose the luminal surface, under sterile conditions. The aortas were then tied tightly at both ends and digested with $0.2 \%$ collagenase I. After incubation at $37^{\circ} \mathrm{C}$ in $5 \% \mathrm{CO}_{2}$ for $1 \mathrm{~h}$, the aortas were washed with medium and cut into pieces; both ends were discarded. The pieces were placed, lumen side down, onto collagen-coated flasks containing DMEM supplemented with 15\% heat-inactivated FBS and penicillin/streptomycin and then incubated at $37^{\circ} \mathrm{C}$ in $5 \% \mathrm{CO}_{2}$. Once the cells had formed a monolayer, the explants were removed, and the cells were subcultured. The cells were identified by their endothelial cell-specific, cobblestone-like morphology, and the purity of the VECs was determined by immunocytochemical staining for Factor VIII. In all experiments, the cells used were passage 3-5.

\section{MTT assay}

VECs were seeded in 96-well-plates $\left(1 \times 10^{5}\right.$ cells $\left./ \mathrm{mL}\right)$ and incubated in DMEM with $15 \% \mathrm{FBS}$ at $37^{\circ} \mathrm{C}$ in $5 \% \mathrm{CO}_{2}$. After treatment with paeonol and ox-LDL, the cells were incubated for $4 \mathrm{~h}$ with fresh medium containing $20 \mu \mathrm{L} \mathrm{MTT}(5 \mathrm{mg} / \mathrm{mL})$. The medium was then removed, and $150 \mu \mathrm{L}$ DMSO was added to each well to dissolve the precipitate. The absorbance of MTT at $490 \mathrm{~nm}$ was detected using a microplate reader (Spectra MaxMze, VT, USA).

\section{Transfection with a miR-21 mimic and inhibitor}

VECs were seeded in 6-well plates and incubated in DMEM with $15 \% \mathrm{FBS}$ at $37^{\circ} \mathrm{C}$ and $5 \% \mathrm{CO}_{2}$. After dilution of $0.6 \mu \mathrm{L}$ miR-21 mimic $(20 \mu \mathrm{mol} / \mathrm{L}$ stock $)$ or $6 \mu \mathrm{L}$ miR-21 inhibitor $(20$ $\mu \mathrm{mol} / \mathrm{L}$ stock) in $400 \mu \mathrm{L}$ culture medium without serum, $12 \mu \mathrm{L}$ HiPerFect transfection reagent was added to the diluted miR$21 \mathrm{mimic} /$ inhibitor and mixed by vortexing to form transfection complexes. The complexes were added drop-wise to the VECs medium, and the plate was gently agitated. The VECs were incubated with the transfection complexes for $24 \mathrm{~h}$, and the medium was then changed as required.

\section{RNA extraction and qRT-PCR assay}

Total RNA was extracted using QIAzol Lysis Reagent for both miR-21 and PTEN mRNA analyses. For detection of gene expression, qRT-PCR was performed using Quantitect SYBR Green PCR Kits according to the manufacturer's protocol. Relative expression was evaluated using the comparative CT method and normalized to the expression of U6 small RNA.

\section{Dual-luciferase reporter assay}

The sequence for miR-21 was obtained from PubMed, and the matched sites are shown below. Luciferase reporter plasmids containing a wild type or mutant 3 '-UTR of PTEN were constructed to verify the putative binding site for miR-21. The wild type or mutant reporter plasmid was cotransfected into VECs, along with the miR-21 mimic. After $36 \mathrm{~h}$, luciferase activity was measured using the dual-luciferase assay system. The firefly luciferase activity of each sample was normalized to the Renilla reniformis luciferase activity.

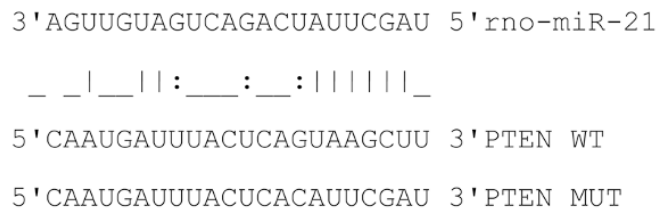




\section{Western blotting}

VECs were lysed in SDS sample buffer, and total protein was extracted from the lysates. Protein concentrations were determined using a BCA Protein Assay kit. The proteins were separated by $10 \%$ SDS-polyacrylamide gel electrophoresis and transferred onto nitrocellulose membranes, which were blocked for $3 \mathrm{~h}$ at room temperature with phosphate buffered saline-Tween containing 5\% nonfat milk. Then, membranes were incubated with antibodies against $\beta$-actin and PTEN. Immunoreactive proteins were visualized by enhanced chemiluminescence detection of secondary antibodies against mouse or rabbit antigens conjugated to horseradish peroxidase.

\section{Statistical analysis}

All data are presented as means \pm SD and were analyzed using SPSS 17.0 (USA). Student's $t$ test was used for statistical comparisons between two groups, and one-way ANOVA was used for multiple groups. A difference was considered statistically significant when $P<0.05$.

\section{Results}

\section{Establishment of injured VECs by exposure to ox-LDL}

VECs were seeded in 96-well plates with DMEM containing $10 \%$ FBS at $1 \times 10^{5}$ cells $/ \mathrm{mL}$ and cultured at $37^{\circ} \mathrm{C}$ in $5 \% \mathrm{CO}_{2}$. After achieving $80 \%$ confluence, the cells were treated with various concentrations of ox-LDL $(5,10,20,40$, or $80 \mathrm{mg} / \mathrm{L})$ for different periods $(12,24$, or $48 \mathrm{~h})$, and cells were then counted using the MTT assay (Figure S1, Figure 2). Ox-LDL treatment inhibited VEC growth in a dose- and time-dependent manner; the growth inhibition rate was close to $50 \%$ when the cells were stimulated with $20 \mathrm{mg} / \mathrm{L}$ ox-LDL for $24 \mathrm{~h}$. These conditions were used as the optimal stimulation concentration and time in subsequent experiments.

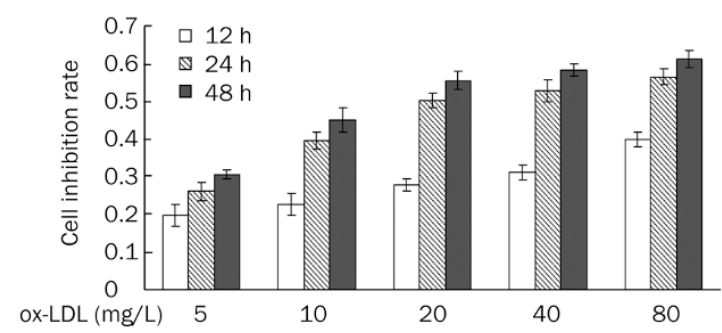

Figure 2. Inhibitory effects of ox-LDL on VECs viability rate were analyzed by MTT assay using a microplate reader at $490 \mathrm{~nm}$. The data were expressed as mean \pm SD. $n=6$. Ox-LDL $(5,10,20,40$, and $80 \mathrm{mg} / \mathrm{L})$ stimulated VECs for 12,24 , and $48 \mathrm{~h}$, respectively.

\section{MiR-21 expression was stimulated in ox-LDL-injured VECs}

The expression of miR-21 in ox-LDL-injured VECs was measured using qRT-PCR. We observed that miR-21 expression was significantly stimulated by treatment with ox-LDL (20 $\mathrm{mg} / \mathrm{L})$ compared with the control group $(P<0.05$, Figure 3$)$, suggesting that miR-21 is involved in ox-LDL-induced VEC damage. Additionally, transfection with a miR-21 mimic

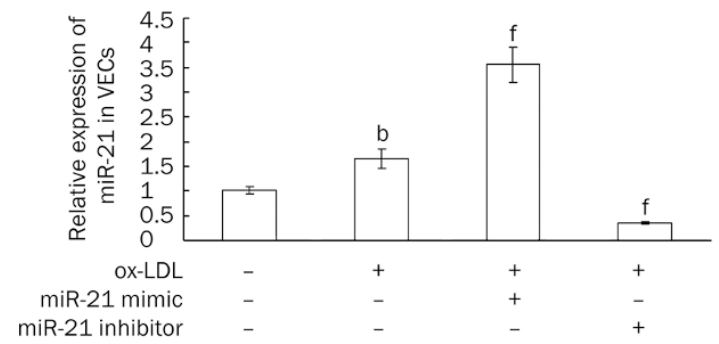

Figure 3. MiR-21 was specifically expressed in ox-LDL-injured VECs. The VECs were transfected with miR-21 mimic or inhibitor, then the cells were treated with ox-LDL $(20 \mathrm{mg} / \mathrm{L})$ for $24 \mathrm{~h}$. All the data came from SYBR Green PCR assay and were expressed as mean \pm SD. $n=3$. ${ }^{b} P<0.05$ vs control group. ${ }^{\mathrm{f}} \mathrm{P}<0.01$ vs ox-LDL group.

significantly enhanced miR-21 expression in VECs, whereas transfection with a miR-21 inhibitor significantly suppressed miR-21 expression $(P<0.01)$ (Figure 3$)$.

\section{PTEN is a target gene of miR-21 in VECs}

To determine whether miR-21 directly targets PTEN in VECs, we constructed luciferase reporter plasmids containing a wild type or mutant $3^{\prime}$-UTR for PTEN. Cotransfection of the wild type reporter plasmid with the miR-21 mimic significantly decreased the relative luciferase activity compared with a miR21 mimic alone $(P<0.01)$, whereas cotransfection with mutant type 3 '-UTR plasmid elicited significantly higher luciferase activity than that seen in the wild type group $(P<0.01)$ (Figure $4 \mathrm{~A})$. In addition, we found that ox-LDL treatment significant reduced the expression levels of PTEN mRNA and protein (Figure 4B and 4C) and that these changes were reversed by treatment with the miR-21 inhibitor. In contrast, a much more significant reduction in expression of PTEN mRNA and protein was observed in the miR-21 mimic-transfected group (Figure 4B and 4C). These findings suggest that PTEN is a downstream target gene of miR-21 in VECs and that miR-21 suppresses PTEN by directly binding to its 3'-UTR.

\section{Paeonol suppresses miR-21 expression in ox-LDL treated VECs}

First, we pretreated VECs with paeonol at various concentrations $(15,30,60,120,240$, or $480 \mu \mathrm{mol} / \mathrm{L})$ for various lengths of time $(6,12,24$, or $48 \mathrm{~h})$. We next treated the cells with 20 $\mathrm{mg} / \mathrm{L}$ ox-LDL for another $24 \mathrm{~h}$. After treatment, cell survival was tested using the MTT assay. It was observed that the cells in paeonol-treated group had a higher survival rate than the ox-LDL group; this effect was dose and time dependent (Figure 5). An optimal condition for paeonol treatment in VECs (120 $\mu \mathrm{mol} / \mathrm{L}$ for $24 \mathrm{~h})$ was established and used in subsequent experiments.

To test whether the protective effect of paeonol is associated with changes in miR-21 expression in VECs, the expression of miR-21 after paeonol and ox-LDL treatment was measured by qRT-PCR. Paeonol treatment significantly decreased oxLDL-induced miR-21 expression in a dose-dependent manner $(P<0.01$, Figure 6$)$, suggesting that the protective effect of 
A

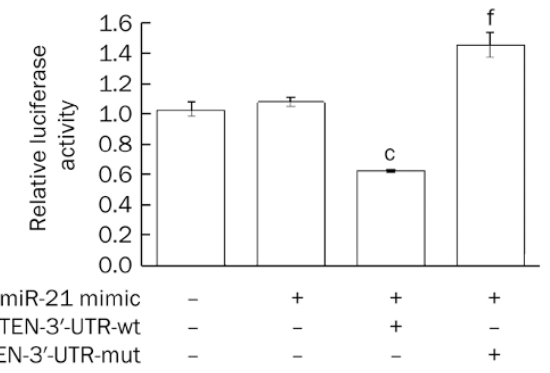

B

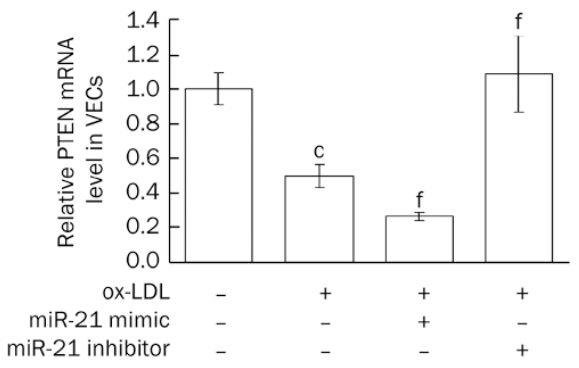

C

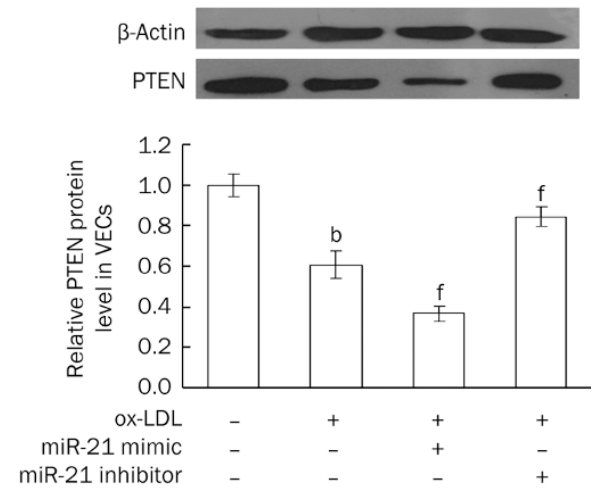

Figure 4. PTEN was a target gene of miR-21 and miR-21 expression was inversely correlated with PTEN expression in ox-LDL stimulated VECs. The data were expressed as mean \pm SD. $n=3$. (A) The wild type or mutant reporter plasmid was cotransfected into VECs with miR-21 mimic. ${ }^{\circ} P<0.01$ vs mimic group. ${ }^{f} P<0.01$ vs $3^{\prime}$ UTR wild type group. (B) Effects of miR-21 mimic and inhibitor on PTEN mRNA expression were measured by SYBR Green PCR assay. ${ }^{c} P<0.01$ vs control group. ${ }^{f} P<0.01$ vs ox-LDL group. (C) Total protein was harvested from cultured VECs and analyzed by Western blotting for PTEN. ${ }^{b} P<0.05,{ }^{c} P<0.01$ vs control group. ${ }^{f} P<0.01$ vs ox-LDL group.

paeonol on VECs was associated with modulation of miR-21 expression.

Paeonol prevents downregulation of PTEN in ox-LDL treated VECs To test whether the protective effect of paeonol in ox-LDLtreated VECs was associated with regulation of PTEN, we treated VECs with $120 \mu \mathrm{mol} / \mathrm{L}$ of paeonol for $24 \mathrm{~h}$, followed by $24 \mathrm{~h}$ of ox-LDL treatment. Ox-LDL treatment significantly suppressed the expression of PTEN at both the mRNA and protein level, and these effects were prevented by pretreat-

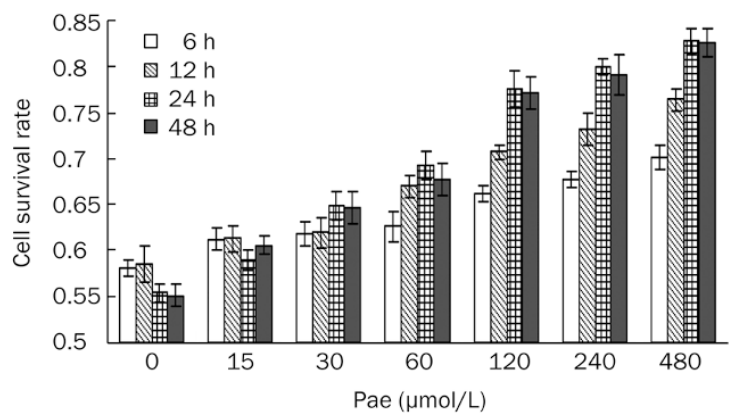

Figure 5. Effects of paeonol on ox-LDL-injured VECs survival rate were analyzed by MTT assay using a microplate reader at $490 \mathrm{~nm}$. The data were expressed as mean \pm SD. $n=6$. VECs were pretreated with different concentrations of paeonol $(15,30,60,120,240$, and $480 \mu \mathrm{mol} / \mathrm{L})$ for $6,12,24$, and $48 \mathrm{~h}$, respectively, then treated with $20 \mathrm{mg} / \mathrm{L}$ ox-LDL for another $24 \mathrm{~h}$.

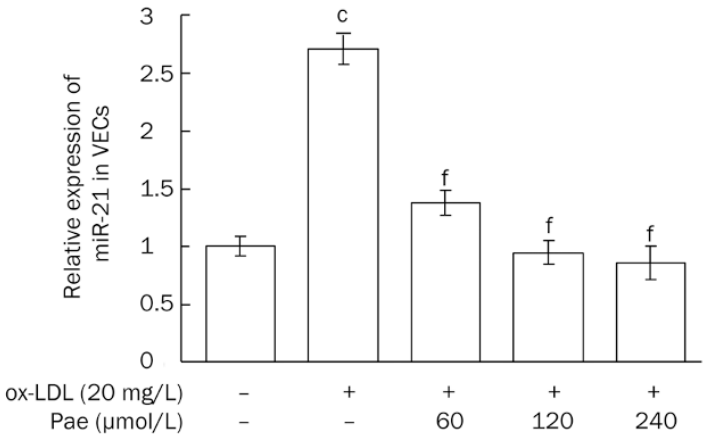

Figure 6. Downregulative effects of paeonol on miR-21 expression were analyzed by SYBR Green PCR assay. VECs were pretreated with varying concentrations of paeonol $(60,120$, and $240 \mu \mathrm{mol} / \mathrm{L})$ for $24 \mathrm{~h}$ and stimulated by ox-LDL $(20 \mathrm{mg} / \mathrm{L})$ for another $24 \mathrm{~h}$, then miR-21 expression was analyzed. Data were expressed as mean \pm SD. $n=3$. ${ }^{\circ} P<0.01$ vs control group. ${ }^{\mathrm{f}} \mathrm{P}<0.01$ vs ox-LDL group.

ment with paeonol (Figure 7A and 7B). In addition, to further characterize the relationship between miR-21 and PTEN in paeonol-treated VECs, the mRNA and protein expression levels of PTEN were assayed in ox-LDL-treated VECs pretreated with paeonol in the presence of the miR-21 mimic or miR-21 inhibitor. We found that the miR-21 mimic rescued the effect of paeonol on the protein expression of PTEN, whereas addition of a miR-21 inhibitor enhanced paeonol-induced increases in PTEN at both the mRNA and protein levels (Figure 7A and 7B). These results suggested that regulation of PTEN by miR21 might be involved in the protective effect of paeonol in oxLDL-induced VEC injury.

\section{Paeonol treatment reduced TNF- $\alpha$ release by ox-LDL treated VECS}

To test whether the effect of paeonol on ox-LDL induced injury in VECs is associated with suppression of TNF-a production, the levels of TNF-a were measured using an ELISA. 
A

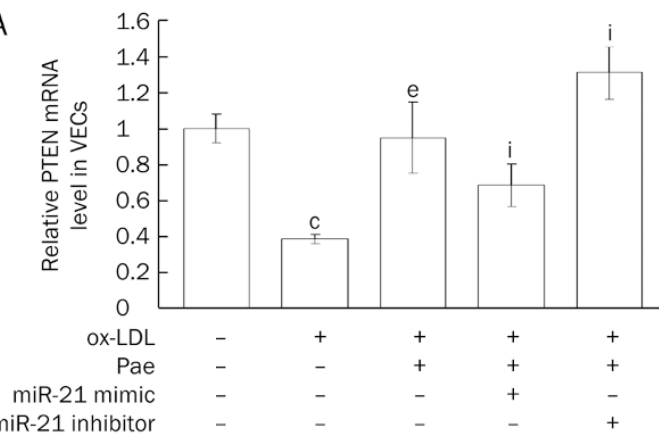

B
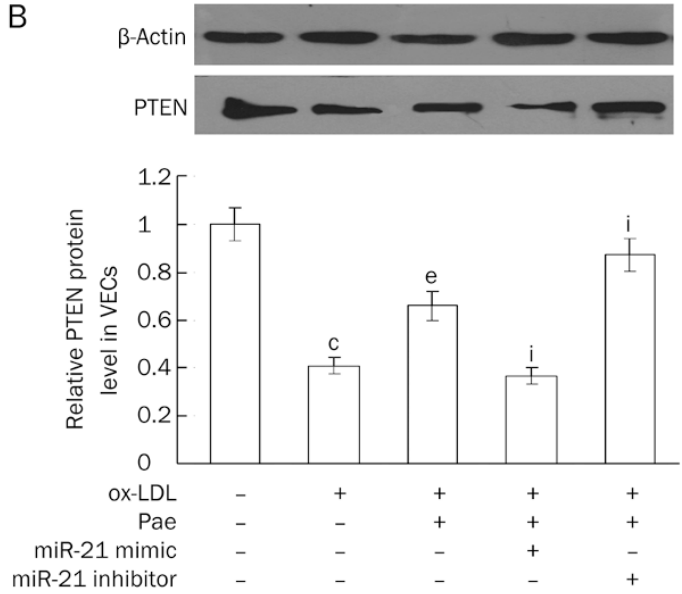

Figure 7. (A) Effects of paeonol on PTEN mRNA level was detected by SYBR Green PCR assay. (B) Effect of paeonol on PTEN protein level was analyzed by Western blotting. MiR-21 mimic or inhibitor was transfected into VECs, then VECs were pretreated with paeonol $(120 \mu \mathrm{mol} / \mathrm{L})$ for $24 \mathrm{~h}$ and stimulated with ox-LDL $(20 \mathrm{mg} / \mathrm{L})$ for another $24 \mathrm{~h}$. Data were expressed as mean \pm SD. $n=3 .{ }^{c} P<0.01$ vs control group. ${ }^{e} P<0.01$ vs oxLDL group. ' $P<0.01$ vs $P$ ae group.

We observed that ox-LDL treatment significantly stimulated TNF-a release in VECs and that this effect was prevented by paeonol pretreatment. Moreover, the effect of paeonol on TNF-a release was partially reversed or significantly enhanced by transfection with miR-21 mimic or inhibitor, respectively (Figure 8). These results suggest that the protective effect of paeonol against ox-LDL induced injury in VECs might be associated with miR-21 mediated suppression of TNF-a release.

\section{Discussion}

Paeonol is a proven anti-atherosclerotic herbal extract with a potent anti-inflammatory activity ${ }^{[11]}$. Our previous investigations have indicated that its anti-atherosclerotic effects are associated with protection of endothelial cells from injury ${ }^{[5,11]}$. However, the underlying mechanisms are still unclear. miRNAs suppress target transcription and translation by binding to the $3^{\prime}$-untranslated regions ( $3^{\prime}$ UTRs) of target mRNAs. These small endogenous silencers can regulate diverse biological processes, such as proliferation, apoptosis,

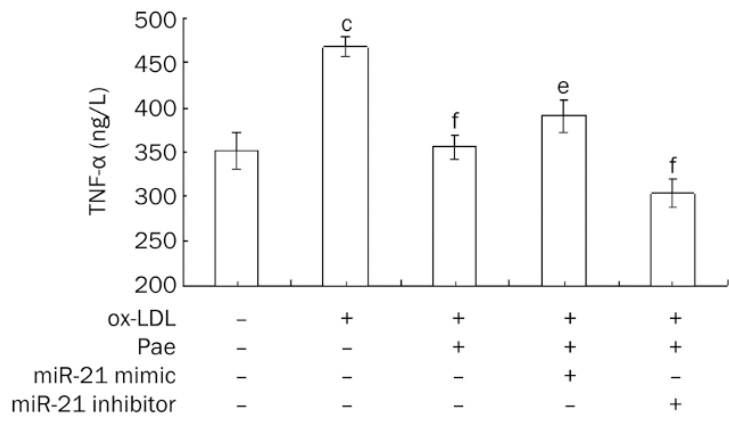

Figure 8. Paeonol inhibited TNF- $\alpha$ expression in ox-LDL induced VECs. MiR-21 mimic or inhibitor was transfected into VECs, then the VECs were pretreated with paeonol $(120 \mu \mathrm{mol} / \mathrm{L})$ for $24 \mathrm{~h}$ and stimulated with oxLDL $(20 \mathrm{mg} / \mathrm{L})$ for another $24 \mathrm{~h}$. TNF- $\alpha$ expression was detected by ELISA assay kit. Data were expressed as mean \pm SD. $n=3 .{ }^{\mathrm{c}} P<0.01$ vs control group. ${ }^{\mathrm{e}} P<0.05,{ }^{\mathrm{f}} P<0.01$ vs ox-LDL group.

angiogenesis, cholesterol metabolism and inflammation ${ }^{[7]}$. Increasing evidence indicates that miRNAs play important roles in the development of $\mathrm{AS}^{[12]}$. miR-21 is highly expressed in vascular endothelial cells ${ }^{[13]}$, cardiomyocytes ${ }^{[14]}$, cardiac fibroblasts ${ }^{[15]}$ and therefore may be critical in the development of cardiovascular disease. In this study, we found that the protective effect of paeonol against ox-LDL VEC injury was at least partially due to its effects on miR-21 expression. Moreover, we observed that pretreatment with paeonol also reversed the suppression of PTEN expression induced by oxLDL. Interestingly, studies have demonstrated that PTEN is a downstream target gene of miR-21 in cancers ${ }^{[16-19]}$, because its $3^{\prime}$-UTR contains 16 base sites that are complementary to the miR-21 sequence ${ }^{[1,20]}$. However, it is unknown whether paeonol-mediated protection of VECS against ox-LDL injury involves miR-21-dependent modulation of PTEN.

In the current study, we found not only that paeonol pretreatment antagonized the suppression of PTEN induced by ox-LDL treatment but also that the expression of PTEN is regulated by miR-21. Additionally, our dual-luciferase reporter assay indicated that miR-21 binds directly to PTEN's 3'UTR, inhibiting PTEN expression. As a result, we suggest that miR21-mediated regulation of PTEN contributes to the protective effects of paeonol,

TNF- $\alpha$ is an important inflammatory factor that is critical in mediating inflammatory diseases such as AS and arthritis ${ }^{[21]}$. In the current study, we found that ox-LDL-induced TNF- $a$ release was prevented by paeonol pretreatment and that transfection with a miR-21 mimic or inhibitor, when combined with paeonol, partially reversed or increased TNF-a release, respectively. Therefore, it is possible that the paeonol's effects on TNF-a release are miR-21-dependent and may contribute to the protection of VECs against ox-LDL-induced cell injury. Nevertheless, the relationship between PTEN and TNF-a release needs further investigation.

Taken these findings together, the current study indicates that the protective effect of paeonol against ox-LDL-induced injury of VECs is at least partially miR-21-dependent. This 
miRNA modulates the downstream target gene, PTEN, as well as TNF-a release. Above all, this study validates miR-21 as a biomarker for vascular inflammation and clarifies the molecular mechanism by which paeonol aids in the treatment of vascular diseases.

\section{Acknowledgements}

The studies were mainly supported by research grants from the National Natural Science Foundation of China (81073090 and 81274134). We thank Prof Hou-kai LI (Shanghai University of Traditional Chinese Medicine) for his contribution to the English translation.

\section{Author contribution}

Ya-rong LIU wrote the paper; Ya-rong LIU and Jun-jun CHEN performed the experiments and analyzed the data; and Min DAI designed the study and supervised the project.

\section{Supplementary materials}

The supplementary figures are available on the web site of APS.

\section{References}

1 Cheng Y, Zhang C. MicroRNA-21 in cardiovascular disease. J Cardiovasc Trans Res 2010; 3: 251-5.

2 Bruyndonckx L, Hoymans VY, Van Craenenbroeck AH, Vissers DK, Vrints CJ, Ramet J, et al. Assessment of endothelial dysfunction in childhood obesity and clinical use. Oxid Med Cell Longev 2013; 2013: 174782.

3 Fu PK, Wu CL, Tsai TH, Hsieh CL. Anti-inflammatory and anticoagulative effects of paeonol on LPS-induced acute lung injury in rats. Evid Based Complement Alternat Med 2012; 2012: 837513.

4 Bao MH, Zhang YW, Zhou HH. Paeonol suppresses oxidized lowdensity lipoprotein induced endothelial cell apoptosis via activation of LOX-1/p38 MAPK/NF-KB pathway. J Ethnopharmacol 2013; 146: 543-51

5 Pan LL, Dai M. Paeonol from Paeonia suffruticosa prevents TNF$\alpha$-induced monocytic cell adhesion to rat aortic endothelial cells by suppression of VCAM-1 expression. Phytomedicine 2009; 16: 1027 32.

6 Chen N, Liu D, Soromou LW, Sun J, Zhong W, Guo W, et al. Paeonol suppresses lipopolysaccharide induced inflammatory cytokines in macrophage cells and protects mice from lethal endotoxin shock.
Fundam Clin Pharmacol 2013; 17.

7 Hata A. Functions of microRNAs in cardiovascular biology and disease. Annu Rev Physiol 2013; 75: 69-93.

8 Kumarswamy R, Volkmann I, Thum T. Regulation and function of miRNA-21 in health and disease. RNA Biol 2011; 8: 706-13.

9 Ji R, Cheng Y, Yue J, Yang J, Liu X, Chen H, et al. MicroRNA expression signature and antisense-mediated depletion reveal an essential role of microRNA in vascular neointimal lesion formation. Circ Res 2007; 100: 1579-88.

10 Pan LL, Dai M, Wang W. A new method for culturing endothelial cells from the rat aorta. Chin Pharmacol Bull 2007; 23: 410-3.

11 Li H, Dai M, Jia W. Paeonol attenuates high-fat-diet-induced atherosclerosis in rabbits by anti-inflammatory activity. Planta Med 2009; 75: $7-11$.

12 Madrigal-Matute J, Rotllan N, Aranda JF, Fernández-Hernando C. MicroRNAs and atherosclerosis. Curr Atheroscler Rep 2013; 15: 322.

13 Weber M, Baker MB, Moore JP, Searles CD. MiR-21 is induced in endothelial cells by sheer stress and modulates apoptosis and eNOS acting. Biochem Biophys Res Commun 2010; 393: 643-8.

14 Cheng Y, Ji R, Yue J, Yang J, Liu X, Chen H, et al. MicroRNAs are aberrantly expressed in hypertrophic heart: do they play a role in cardiac hypertrophy? Am J Pathol 2007; 170: 1831-40.

15 Roy S, Khanna S, Hussain SR, Biswas S, Azad A, Rink C, et al. MicroRNA expression in response to murine myocardial infarction: miR-21 regulates fibroblast metalloprotease-2 via phosphatase and tensin homologue. Cardiovasc Res 2009; 82: 21-9.

16 Xiong B, Cheng Y, Ma L, Zhang C. MiR-21 regulates biological behavior through the PTEN/PI-3K/Akt signaling pathway in human colorectal cancer cells. Int J Oncol 2013; 42: 219-28.

17 Zhang BG, Li JF, Yu BQ, Zhu ZG, Liu BY, Yan M. microRNA-21 promotes tumor proliferation and invasion in gastric cancer by targeting PTEN. Oncol Rep 2012; 27: 1019-26.

18 Liu CZ, Liu W, Zheng Y, Su JM, Li JJ, Yu L, et al. PTEN and PDCD4 are bona fide targets of microRNA-21 in human cholangiocarcinoma. Chin Med Sci J 2012; 6: 65-72.

19 Qin X, Yan L, Zhao X, Li C, Fu Y. microRNA-21 overexpression contributes to cell proliferation by targeting PTEN in endometrioid endometrial cancer. Oncol Lett 2012; 4: 1290-6.

20 Salmena L, Carracedo A, Pandolfi PP. Tenets of PTEN tumor suppression. Cell 2008; 133: 403-14.

21 Ragino YI, Chernyavskiy AM, Tsimbal SY, Shcherbakova LV, Polonskaya YV, Kashtanova EV. Relationship of blood levels of inflammatory and destructive biomarkers in coronary atherosclerosis with long-term results of surgical revascularization. Bull Exp Biol Med 2013; 155: 314-7. 